\section{Buchrezension zu:}

\section{Toxic}

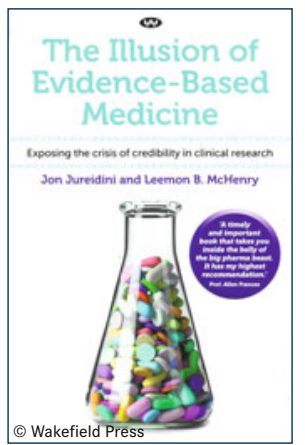

The Illusion of Evidence-Based

Medicine

Exposing the Crisis of

Credibility in Clinical Research

Jon Jureidini und Leemon B.

McHenry

318 S., Wakefield Press, 2020. SC, 29,80€.

ISBN: 9781743057247

Auch als E-Book erhältlich

DOI: $10.1007 / \mathrm{s} 12268-020-1476-1$

(C) Der Autor 2020

Die Grundlagenforschung wird in weiten Teilen durch öffentliche Geldgeber und Stiftungen finanziert. Ganz anders sieht es mit der klinischen Forschung aus. In den allermeisten Fällen werden klinische Studien über neue Arzneistoffe von den Entwicklern des
Arzneistoffs finanziert. Und darin liegt das Grundproblem: Vielfältige Interessenkonflikte machen es wahrscheinlich, dass neue Arzneistoffe nicht wirklich objektiv untersucht werden. Einige im vorliegenden Buch belegte Beispiele: „Wichtige“ Kliniker werden mit finanziellen incentives als „Meinungsführer" gewonnen, Dosierungen von Vergleichsarzneistoffen werden bewusst zu niedrig (geringe Wirkung) oder zu hoch angesetzt (mehr unerwünschte Wirkungen), Publikationen werden marketinggerecht von ghostwriting companies verfasst und therapeutische Leitlinien werden von Klinikern mitbestimmt, die finanziell mit Pharmafirmen verbandelt sind.

Diese und andere Probleme der klinischen Forschung exponieren die Autoren des Buchs am Beispiel von zwei klassischen klinischen Studien über den Einsatz von Serotonin-WiederaufnahmeInhibitoren (SSRI) bei Kindern und Jugendlichen. Die Studien waren für die Pharmafirmen von großer finanzieller Bedeutung, weil es darum ging, die SSRI Paroxetin und Citalopram aus dem off-labe/-
Bereich in den offiziellen Verschreibungsbereich zu bringen. Mit großer Akribie stellen die Autoren dar, wie Daten geschönt wurden, unerwünschte Wirkungen verschwiegen und führende Kliniker ohne wirkliche wissenschaftliche Beiträge zu honory authors auf Publikationen in prestigeträchtigen Journalen wurden. Die Autoren zeigen auch sehr gut, dass wissenschaftliche Journale, Universitäten und wissenschaftliche Fachgesellschaften in das System finanzieller Abhängigkeiten von der Pharmaindustrie involviert sind.

Das liest sich alles recht ernüchternd, entspricht aber in weiten Teilen der Realität. Die Autoren wollen das Buch jedoch nicht so negativ enden lassen und zeigen am Ende Perspektiven auf, wie klinische Forschung langfristig besser gemacht werden kann, für alle Beteiligten. Dabei verweisen sie auf Ansätze des Wissenschaftsphilosophen Karl Popper. Das Buch wird durch einen ausführlichen Anhang von Korrespondenz mit Wissenschaftlern und Journalen sowie ein detailliertes Literaturverzeichnis abgerun- det. Es ist ein must-read für jeden Wissenschaftler, der in irgendeiner Form mit klinischen Studien zu tun hat, um inn für Interessenkonflikte zu sensibilisieren.

Roland Seifert

Medizinische Hochschule Hannover,

Seifert.Roland@mh-hannover.de

* Funding Open Access funding enabled and organized by Projekt DEAL.

* Open Access Dieser Artikel wird unter der Creative Commons Namensnennun 4.0 International Lizenz veröffentlicht, welche die Nutzung, Vervielfältigung, Bearbeitung, Verbreitung und Wiedergabe in jeglichem Medium und Format erlaubt, sofern Sie den/die ursprünglichen Autor(en) und die Quelle ordnungsgemäß nennen, einen Link zur Creative Commons Lizenz beifügen und angeben, ob Änderungen vorgenommen wurden. Die in diesem Artikel enthaltenen Bilder und sonstiges Drittmaterial unterliegen ebenfalls der genannten Creative Commons Lizenz, sofern sich aus der Abbildungslegende nichts anderes ergibt. Sofern das betreffend Material nicht unter der genannten Creative Commons Lizenz steht und die betreffende Handlung nicht nach gesetzlichen Vorschriften erlaubt ist, ist für die oben aufgeführten Weiterverwendungen des Materials die Einwilligung des jeweiligen Rechteinhabers einzuholen. Weitere Details zur Lizenz entnehmen Sie bitte der Lizenzinformation auf http://creativecommons.org/ licenses/by/4.0/deed.de. 